\title{
Guest editorial special issue on intelligent multimedia computing
}

\author{
Ibrahiem El Emary • Aboul-Ella Hassanien
}

Published online: 2 December 2008

(C) Springer Science+Business Media, LLC 2008

\section{Introduction}

Intelligent systems (IS) are an emerging field that consists of complementary elements of fuzzy logic, neural computing, evolutionary computation, machine learning and probabilistic reasoning. Due to their strong learning and cognitive ability and good tolerance of uncertainty and imprecision, soft computing techniques have found wide applications. Needless to say, multimedia data (video, image, audio, text, color, etc.) is one of these applications. In the last few years we have witnessed an explosive growth of multimedia computing, communication and applications. The few key technologies interact in an interesting and yet useful way, bringing along the multimedia revolution. This revolution is transforming the way people live, work, and interacts with each other, and is impacting the way businesses, government services; education, entertainment, and health care are operating. Nowadays popularity of multimedia demands efficient and intelligent strategies in order to cope with the large amount of multimedia data. Combining the arts, science, artificial intelligence, computer science, and the humanities, intelligent multimedia computing has the potential to create significant advances in communication technologies. Human communication is intrinsically multimodal and since advances in technology offers

I. El Emary ( $\varangle)$

Faculty of Engineering, Al Ahliyya Amman University, Amman,

Jordan

e-mail: omary57@hotmail.com

A.-E. Hassanien

Faculty of Computer \& Information, Cairo University, Giza,

Egypt

e-mail: aboitcairo@gmail.com solutions to today's increasing demand for current\& emerging technologies, modern communication systems will also become more multimodal. Intelligent multimedia computing is a very important scientific research domain with a broadening range of applications including applications included games, pictorial encyclopedias, animated science adventures, and interactive story books. Today, interactive computer-based training and corporate communications can be distributed via wide-area network or satellite broadcast for up to the minute accuracy. Interactive intelligent multimedia can reduce training costs while increasing comprehension. The development of new insights and applications results from both fundamental scientific research and the development of new technologies. One of these emerging technologies is computational intelligence, which is a generic term for a specific collection of tools to model uncertainty, imprecision, evolutionary behavior and complex models. We received 23 papers, of which 7 were accepted for publication. The topics covered in this issue cover a wide range of research areas of computational intelligence in multimedia computing including content-based image retrieval, constructed networks of knowledge, intelligent video authentication, designing resilient networks, multicommodity/multimodal international freight simultaneous transportation network equilibrium model, colour quantization and autonomous systems.

\section{Scanning through the issue}

Video authentication is a very challenging problem and of high importance in several applications such as presenting video evidence in the court of law and video surveillance. In several law enforcement applications such as surveillance and forensics, video is often presented as evidence. It is 
therefore of paramount importance to establish the authenticity and reliability of the video data. Richa Singh et al. [1] presents an intelligent video authentication algorithm which integrates learning based Support Vector Machine (SVM) classification with Singular Value Decomposition (SVD) watermarking, which can detect multiple video tampering attacks. The video authentication algorithm embeds the inherent video information in the frames using SVD watermarking and uses it for classification by projecting them into a non-linear SVM hyperplane. The algorithm is validated on an extensive video database containing 3190 tampered and non-tampered videos. Their results show that the proposed algorithm yields very high classification accuracy with low computational complexity.

Colour quantization is a common image processing technique that allows the representation of true colour images using only a small number of colours and is useful for displaying images on limited hardware such as mobile devices, for image compression, and for other applications such as image retrieval. The choice of a good palette is therefore crucial as it directly determines the quality of the resulting image. Colour quantization can also be seen as a clustering problem where the task is to identify those clusters that best represent the colours in an image. Schaefer and Zhou [2] investigate the performance of various fuzzy c-means clustering algorithms for colour quantization of images. In particular, they use Conventional fuzzy c-means as well as some more efficient variants thereof, namely fast fuzzy c-means with random sampling, fast generalized fuzzy c-means, and a recently introduced anisotropic mean shift based fuzzy cmeans algorithm. Experimental results have revealed that fuzzy clustering based colour quantization is able to achieve significantly improved image quality compared to the other techniques. Furthermore, it was shown that the computationally faster variants of fuzzy c-means provide virtually the same image quality as their full conventional counterpart and therefore represent efficient and effective techniques for colour quantization.

A core goal for autonomous systems such as the work proposed by Kevin et al. [3] is automated collaboration in order to perform tasks or share information. The system is always distributed by default and frequently on a largescale. It can be argued that robustness and economy demand the deployment of a tested autonomic supporting infrastructure whenever possible. A knowledge network is a generic structure that organizes distributed knowledge of any format into a system that will allow it to be retrieved efficiently. The rationale of the knowledge network is to act as a middle layer that connects to a multitude of sources, organizes them based on various concepts and finally provides well-structured, pre-organized knowledge to individual services and applications. To use the knowledge network we need a querying mechanism to be able to retrieve information. The knowledge network will organize itself in an autonomous manner and it is possible to use the querying mechanism also as part of the knowledge organization mechanism, to autonomously create temporary views that reflect the use of the system. Kevin et al. [3] paper is an attempt to investigate the peculiarities of node behavior in traversing such a knowledge network. They investigate a variety of methods of traversing a knowledge network and they found that the method of placing edge servers in the network through which application level relay nodes process data before forwarding to each application ultimately reducing the amount of data sent through the network through an intelligent aggregation process was the most efficient method.

The prediction of multi-commodity freight flows on a multimodal network has attracted substantial interest in recent years. The prediction of passenger flows on multimodal urban transportation networks has been studied extensively, and many of the research results have been applied at the practical. However, the study of freight flows at the national, regional and international levels, perhaps owing to inherent difficulties and complexities, has received less attention. Hasan [4] presented an implementation of the International Freight Simultaneous Transportation Equilibrium Model (IFSTEM) that developed in United Nations Economic and Social Commission for Western Asia (ESCWA) to the goods trade through the ports and lands of Jordon, Syria, and Lebanon. Although some socio-economic variables, which are not available, were required for IFSTEM model calibration, some reasonable assumptions were made and it was good enough to draw the following main findings: the IFSTEM model was able to replicate the observed path and O-D pair goods flows for year 2001 through its initial solution; the IFSTEM final solution suggested that the path distribution for most observed O-D pairs flows is not optimal due to the exporters depend only one some measure of attractiveness in their path choice and it should be redistributed to have a great saving in the total freight cost; the IFSTEM can be consider as a good decision support tool that is able to evaluate the value of any scenario that can be reflected through any change in the costs and/or times of its link cost function or any change in the socio-economic variables, as the case of year 2007 prediction.

Content-based image retrieval (CBIR) is an attractive research area due to the rapid growth in image databases in many domains, like medical image management, multimedia libraries, document archives, art collections, geographical information system, law enforcement agencies, and journalism. Finding an image from a large set of images is an extremely difficult problem. One solution is to label images manually, but this is very expensive, time consuming and infeasible for many applications. Furthermore, the labeling process depends on the semantic accuracy in describing the image. Therefore much Content based Image Retrieval (CBIR) systems are developed to extract low-level 
features for describing the image content. However, this approach decreases the human interaction with the system due to the semantic gap between low-level features and highlevel concepts. Aboulmagd et al. [5] propose a fuzzy logic approach to narrow the gap between the quantitative similarity evaluated by the system and the qualitative evaluation offered by the user to calculate the query relevance. They use Fuzzy Attributed Relational Graphs FARGs to represent images with modifications in the image feature representation in a way that uses the fuzzy set and fuzzy logic concepts to express the middle level of image content. They propose a graph matching algorithm that simulates the way the humans think when comparing images. In their study, they attempt to verify how fuzzy concept helps in narrowing the gap between low level features and high level concepts and is able to model the image objects and its attributes and spatial relation. The proposed system is evaluated by different users with different perspectives and is found to match users' satisfaction to a high degree.

The main reason why neural networks (NN's) are perceived as a critical core technology for Intelligent Multimedia Computing based on their its capability of learning, which enables machines to be taught to interpret possible variations of the same object or pattern, e.g., scale, orientation, and perspective. More specifically, NN's have the following attributes: (1) It offers unsupervised clustering (i.e., no specific target label/response is provided for any input) and/or supervised learning mechanisms (the input and corresponding target label/response are both given) for recognition of objects that are deformed or with incomplete information. Ultimately, a neural information engine can be "trained" to see or hear, to recognize objects or speech, or to perceive human gestures. (2) Neural networks are powerful pattern classifiers and encompass many similarities with statistical pattern recognition approaches. They appear to be most powerful and appealing when explicit a priori knowledge of underlying probability distributions is unknown. By their training by example property, neural network classifiers may be properly trained to provide outputs that none parametrically approximate a posteriori class probabilities; and (3) It offers a universal approximation capability, i.e., they are able accurately to approximate unknown systems based on sparse sets of noisy data. In this context, some neural models have also effectively incorporated statistical signal-processing and optimization techniques. One of the NN's systems that are covered in this issue is Probabilistic Neural Networks (PNN) which represents some kind of supervised neural network, proposed by Specht as an alternative to back-propagation neural network. The key advantages of PNN are that, training requires only a single pass, and decision surfaces are guaranteed to approach the Bayesoptimal decision boundaries, as the number of training samples grows. Furthermore, shape of the decision surface can be made as complex as necessary, or as simple as desired, by choosing an appropriate value of the smoothing parameter; erroneous samples can be tolerated, and sparse samples are adequate for network performance. Ramakrishnan and El Emary [6] reviews the PNN, modified PNN, various learning approaches employed to train the PNN and some comparisons various types of PNN. Experimental results have been carried out and it verify the ability of modified PNN in achieving good classification rate in compared with traditional PNN or back propagation neural network BPNN and KNN.

Reliability has been a traditional goal within telecommunication network design. There has been some recent research interest in developing algorithms for problems of guaranteeing resilience against failures. Due to the fact that the assurance of continuity in traffic is a vital demand in today's networks, we have to be able to provide backup paths at the moment a failure on a edge (or in multiple edges) on the primary path occurs. For this, the backup path has to be built a priory. Usually, fault tolerance is achieved by providing backup paths, which are used in case of an edge failure on a primary path. Grosan et al. [7] proposes an algorithm dealing with multi-objectivity in resilience network that are fault tolerant against link failures. The authors consider this task as a multi-objective optimization problem: to provide resilience in networks while minimizing the cost subject to capacity constraint. They propose a stochastic approach, which can generate multiple Pareto solutions in a single run. The feasibility of the proposed method is illustrated by considering several network design problems using a single weighted average of objectives and a direct multiobjective optimization approach using the Pareto dominance concept. The performance of the proposed approach is tested for three different networks and the approach is able to detect multiple feasible solutions in a very short time (less than a second).

The editors wish to thank the referees who have critically evaluated the papers within the short stipulated time. Finally, we hope the reader will share our joy and find this special issue very useful. We would like to take this opportunity to thank Professor Bezalel Gavish, Editor-in-chief, Journal of Telecommunication Systems for all the timely advices and help and also for the opportunity for editing this important scientific work.

\section{References}

1. Singh, R., Vatsa, M., Sanjay, K., \& Upadhyay, S. S. Integrating SVM classification with SVD watermarking for intelligent video authentication.

2. Schaefer, G., \& Zhou, H. Fuzzy clustering for colour reduction in images.

3. Curran, K., Baumgarten, M., Mulvenna, M., Nugent, C., \& Greer, K. A computational intelligence method for traversing dynamically constructed networks of knowledge. 
4. Hasan, M. K. Multimodal and multicommodity international freight simultaneous transportation network equilibrium model.

5. Aboulmagd, H., El-Gayar, N., \& Onsi, H. A new approach in content-based image retrieval using fuzzy logic.

6. Ramakrishnan, S., \& El Emary, I. M. M. Comparative study between traditional and modified probabilistic neural networks.

7. Grosan, C., Abraham, A., \& Hassainen, A. E. Designing resilient networks using multicriteria metaheuristics.

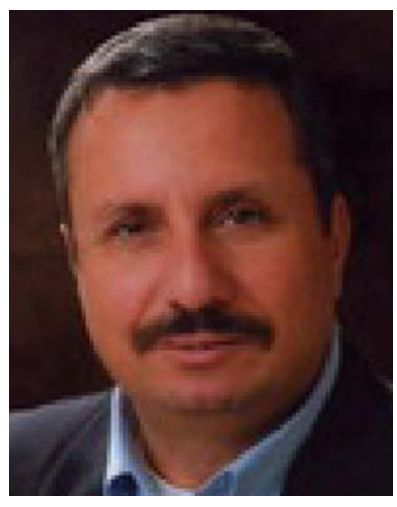

Ibrahiem El Emary received the Dr. Eng. Degree in 1998 from the Electronic and Communication Department, Faculty of Engineering, Ain Shams University, Egypt. He is a visiting Associate Professor and head of computer engineering department at Al Ahliyya Amman University, Amman, Jordan. His research interests cover: analyzing the various types of analytic and discrete event simulation techniques, performance evaluation of communication networks, application of intelligent techniques in managing computer communication network, and performing comparative studies between various policies and strategies of routing, congestion control, sub netting of computer communication networks. He published more than 70 articles in various refereed international journals and conferences covering: Computer Networks, Artificial Intelligent, Expert
Systems, Software Agents, Information Retrieval, E-learning, Case Based Reasoning, Image processing, wireless sensor networks and Pattern Recognition. Also, in the current time, he is too interested in making a lot of scientific research in wireless sensor networks in view point of enhancing its algorithms of congestion control as well as routing protocols. Also, he is an editor in chief of two international journals and associate editor of more than 10 international journals.

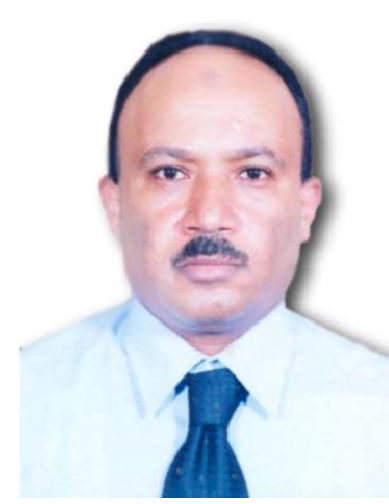

Aboul-Ella Hassanien is an associated professor at Cairo University, Faculty of Computer and Information Technology Department. He has involved in the organized/Scientific committees member of many international conferences. He also serves the editorial board of some reputed International journals and has guested edited for many special issues on various topics related to computational intelligence and soft computing. He has authored/co-authored several refereed journal/conference papers and book chapters. He has published many papers in prestigious Journals. He has received the excellence younger researcher award from Kuwait University for the academic year 2003/2004. His research interests include, rough set theory, wavelet theory, X-ray Mammogram analysis, fuzzy image processing and multimedia data mining. Homepage: http://www.softcomputing.net/ abo. 